\title{
Entwicklungsmechanik und praktische Medizin.
}

Von Dr. Georg Ettisch in Berlin-Dahlem;

(früher Ober-Assistent am Anatomischen Institut in Halle).

I.

Seit etwa 40 Jahren ist das große Gebiet der biologischen Naturwissenschaften um eine Diszipliff' 'erweiterf, die für Theorie wie Praxis, sodann aber auch in wissenschaftshistorischer Beziehung von höchster Bedeutung ist, ohne daß ihr indessen bisher in Deutschland eine dieser Bedeutung entsprechende Verbreitung und $\mathrm{An}$ erkennung beschieden war. Trotz dieser ihrer verhältnismäßigen Jugend verfügt aber die "Entwicklungsmechanik" oder "Kausale Morphologie" bereits über einen Schatz von Ergebnissen, der gerade für den Arzt eine Fundgrube der wichtigsten Anregungen und Hinweise darstellt. Dennoch ist sie in ihrem Wesen und ihren $\mathrm{Er}$ gebnissen den meisten Praktikern oft kaum dem Namen nach bekannt, und selbst unter den Theoretikern finden sich nicht wenige, die mit ihrem Namen einen völlig unzureichenden Begriff verbinden.

Dieser Aufsatz setzt es sich zum Ziele, zur Kenntnis ihres Wesens beizutragen. Es kann sich auf Wunsch des Herausgebers dieser Wochenschrift hier allein darum handeln, dem biologisch interessierten Arzt, der sich in Ausübung seines Berufes allmählich von der Theorie entfernt hat, einen Begriff $\mathrm{zu}$ geben von der Art der entwicklungsmechanischen Wissenschaft (Em.). Ihre Methoden sowie ihre wesentlichsten Lehren werden kurz darzulegen und an charakteristischen Beispielen zu erörtern sein. Aber auch dabei kann es sich allein um eine Auswahl handeln, da ein Eingehen auf alle Zweige dieser Wissenschaft ebenfalls den Rahmen dieses Aufsatzes überschreiten würde.

Die „Kausale Morphologie“ wurde ins Leben gerufen von einem Manne, der von Beginn seiner überaus reichen und bedeutungsvollen wissenschaftlichen Tätigkeit an ein Führer zu neuen Zielen war Wilhelm Roux ist ihr Begründer. In seiner Habilitationsschrift: „Ueber die Bedeutung der Deszendenzlehre" 1880 finden sich bereits die ersten Grundgedanken, die zu einer neuen Wissenschaft führen sollten. Einen weiteren Baustein dazu trug er in seinem berühmten "Kampf der Teile im Organismus" herbei. Im Jahre 1886 tauchte das" erstemal der Name „Entwicklungsmechanik“ oder „,Kausale Morphologie" auf. Ro u x "Erbeitete seiner Wissenschaft" auch die Methoden aus, bestimmte ihre wesentlichsten Begriffe und schenkte ihr dazu auch noch die ersten fundamentalen, positiven $\mathrm{Er}$ gebnisse. Er begründete fernerhin eine besondere Zeitschrift für sein Forschungsgebiet, das Arch. f. Entw. Mech., das er heute noch selbst herausgibt. Neben dieser Zeitschrift, die der speziellen Einzelforschung dient, erscheinen seine „Aufsätze und Vorträge“, in denen Zusammenfassungen und wichtige neue theoretische Abhandlungen zur Veröffentlichung gelangen.

Der Gegenstand der Em. ist die Form des Organismus oder seiner Teile bei Tier und Pflanze. In der Anatomie - der makroskopischen wie auch mikroskopischen - besitzen wir indes schon eine zum Teil jahrtausendealte Morphologie. Von dieser altehr würdigen Wissenschaft unterscheidet sich unsere junge prinzipiell durch ihre Ziele sowie ihre Methode. Dabei verdrängt sie jene alte Morphologie keineswegs, macht sie durchaus nicht überflüssig. Vielmehr bilden gerade deren riesiges gesichertes - soweit man bei einer Naturwissenschaft davon sprechen kann - Tatsachenmaterial und deren noch heute und späterhin zutage zu fördernde Ergebnisse erst die Grundlage, die Anregung und Leitung für die kausale Problemstellung. Aber nicht nur dieses, die bisherige Morphologie ist auch ständig der. Prüfstein für die Theorien, Arbeitshypothesen und auch Ergebnisse der neuen. So darf der Em. keineswegs den Anatomen alter Observanz mit seinen Arbeiten übersehen, will er nicht in der Folge schwer dadurch gestraft werden. Es besteht also notwendigerweise ein inniges Wechselverhältnis zwischen beiden Disziplinen. Beide arbeiten an demselben Gegenstande, nur ist ihr Standpunkt verschieden und, wie schon erwähnt, ihre Methode.

Die bisherige Morphologie, die auch die deskriptive genannt wird, befaßt sich mit der elementaren Analyse des Organismus und seiner Teile, Durch mehr oder weniger komplizierte Techniken, die aber doch alle den Grundcharakter des Zergliederns bewahren, sucht sie die Bauelemente der Organe, Gewebe und Zellen zu erfassen. Auf dem Wege der Beschreibung und Klassifikation wirc das Gefundene dann dargestellt. Solange keine anderen physikalischen Hilfsmittel vorhanden waren, geschah dies durch einfache makroskopische Zergliederung, mit der Erfindung des Mikroskops, der Entdeckung neuer physikalischer Erscheinungen (Polarisation, Doppelbrechung) und dem Ausbau der chemischen Wissenschaft hat auch die Morphologie ihre Hilfsmittel zu jenem obigen Zwecke erweitert und ihren Schatz der Ergebnisse damit um vieles Wertvolle vermehrt. Stets aber liegen die Elemente noch innerhalb des mit bewaffnetem oder unbewaffnetem Auge Wahrnehmbaren. Dennoch würde man der deskriptiven $M$. nicht völlig gerecht, wollte man ihr Wesen allein mit jener einfachen Zergliederung und Beschreibung charkterisieren. Der Anatom begnügte sich z. B. keineswegs mit der Beschreibung der Gelenkformen, vielmehr erforschte er in umfangreichem Maße die Bewegungen, die in diesen Gelenken möglich waren, und erforschte weiter die Gelenkbewegungen mit Rücksicht auf die die Bewegung veranlassenden Muskeln. Der Anatom begnügte sich fêrnerhin nicht mit der Feststellung der bez. Knochenformen und dimensionen, sondern ermittelte auch die Ursache ihres 
Wachstums. Der Histologe beschrieb nicht nur die in den Samenkanälchen des Hodens an denl verschiedensten Stellen sich gerade vorfindenden Elemente, vielmehr brachte er einen Zusammenhang in die vielen verschiedenen Erscheinungsformen der Zellen an diesem Orte und stellte somit den Vorgang der Spermatogenese fest. Er beschrieb fernerhin night nur die vielartigen Zellen, die an den drüsenartigen Organen sich finden, vielmehr erforschte er auch alle diejenigen Vorgänge und Zusammenhänge im Innern der Zelle, die zur Sekretbildung und Sekretausstoßung führen. Der Embryologe beschrieb nicht nur seine gerade gefundenen Resultate, sondern er suchte durch lückenlose Reihenuntersuchung über den h is to ris ch en Verlauf der Organbildung usw. usw. sich Aufklärung zu verschaffen. So sehen wir auch in der sog. deskriptiven Morphologie neben der Zergliederung bei den meisten Forschern auch das Bestreben nach Svnthese, den Zusammenhang unter den Elementen bei Berücksichtigung der Physiologie, der Funktion des gerade zu Erforschenden, aufzufinden, sehen neb̈en der bloßen Beschreibung des bei der elementaren Analyse Gefundenen' auch schon die Bemühung, $\mathrm{Zu}$ sammenhänge und Verläufe historischer Art darzustellen und so zı einer Art von Erklärung zu gelangen.

Die Entwicklungsmechanik dagegen stellt sich eine ganz andere Aufgabe. Sie fragt nicht nach dem "Wi e" der Form, des Baues, sondern prinzipiell nach dem ,W ar um", sucht also die Ursachen festzustellen, die zu der bestimmten Form geführt haben. Daher der Beiname "kausale Morphologie“. Thr liegt das geistige Bedürfnis zugrunde, eine Erscheinung in den Zusammenhang von Ursache und Folge zu bringen; denn erst dann tst sie uns vollkommen verständlich. Die Kenntnis ihrer kleinstert (deskriptiv ermiftelten) Einzelheiten ist dabei natürlich ebenifalls notwendig, reicht aber zı einer vollkommenen kausalen Erkenntnis nicht hin, auch genügt dazu nicht das Wissen um das rein historische Werden jener in Betracht kommenden Erscheinung. So genügt die sog. deskriptive Morphologie wohl unserem Verlangen nach Kenntnis der Erscheinungen des Baues von Organismus oder seiner Teile. Die Em. dagegen, indem sie auf jener Kenntnis fußt, vermag uns Erkenntnis über jenen Gegenstand zu bringen. Was anderes aber heißt Erkenntnis schaffen über Erscheinungen, als sie einordnen in den ursächlichen Zusammenhang alles Geschehens, als: ihre bedingenden Ursachen aufdecken? Ein Beispiel hierzu. Die Embryologie gab uns in C. Rabls Werk eine mustergültige Darstellung von "Bau und Entwicklung der Linse". Dabei aber blieb die Frage unbeantwortet, was die Ursache der Bildung dieses Derivats des äußeren Keimblattes sei, wovon ihr Entstehen, ihre Größe usw. eigentlich abhängt. In einer Reihe von Arbeiten ermittelten entwicklungsmechanische Forscher, daß in jenem Teil der embryonalen Augenanlage (primärer Augenbecher), aus dem dereinst die Netzhaut gebildet wird, die Ursache zur Linsenproduktion liegt. Legt sich dieser. Teil des Augenbechers nicht an das Ektoderm an, oder unterbleibt gar seine Bildung, so liefert das äußere Keimblatt auch keine Linse. Legt sich das Retinablatt des Augenbechers nur in geringer Ausdehnung an das Ektoderm an, so entsteht eine entsprechend verkleinerte Linse. Eine ganze Reihe abnormer Bildungen an diesem Teil des Sehorganes ist neben der normalen so in den Bereich unseres Verständnisses gerückt. Vorher gab es hier einzig ihre Konstatierung und Beschreibung. In ihrem entwicklungsmechanischen Zweige ist daher die Morphologie zu einer Wissenschaft geworden, die den am höchsten entwickelten anorganischen Naturwissenschaften. der Physik und der Chemie, um ein Bedeutendes nähergerückt ist.

Die bisherige Morphologie beschreibt $Z$ ustände, sichtbare Befunde. Aus der Erkenntnis heraus aber, daß alles Organische wird, hat auch die deskriptive Richtung versucht, mit ihren Mitteln dieses Werden zil erfassen. Sie kann diesem Ziel nur dadurch nahekommen, da $B$ sie aus dem.. Werde"gang - etwa eines Organes - möglichst. lückenlos aufeinanderfolgende Stadien der Ausgestaltung herausgreift und beschreibt. So vermag sie in der Tat das historische Werden zu erfassen, das sicherlich als eine Art der Betrachtung des Werdens anerkannt werden mußB. Allein dieses Werden ist völlig beziehungslos, die einzelnen beschriebenen Stadien liegen dennoch isoliert aufeinander wie die Blätter eines Papierstoßes. Aus dem kontinuierlichen Prozeß des Werdens wird eine Folge von verhältnismäBig groben Diskontinuitäten gemacht. Es bleiben daher die zwei wesentlichsten Fragen hier unbeantwortet: 1. warum die $\mathrm{Zu}$ stände gerade in der und keiner anderen Weise aufeinanderfolgen, und 2. ob nicht in der Entstehung und Gestaltung der Teile eine gegenseitige Abhängigkeit hesteht - neben noch anderen wichtigen Fragen. Ein Beispiel mag dies erläutern. Die deskriptive Morphologie gab uns ein vollkommenes Bild von dem historischen Werden des Auges und seiner Teile, etwa der Kornea. Die Em. dagegen vermochte zu zeigen, wie jene Korneabildung abhängt von der des Augenbechers. Exstirpierte man (bei Rana palustris) die Augenanlage, oder unterblieb die Augenbecherbildung, so formte sich auch keine Kornea aus der äußeren Haut. Verpflanzte man dagegen den Augenbecher unter eine beliebige Stelle der Körperhaut, etwa am Bauche, so entstand hier aus der pigmentierten Haut eine Hornhaut. An der ursprünglichen Stelle der Korneabildung unterschied sich die Haut dann in nichts von der der anderen, soweit sie den Körper bedeckte. Die Hornhaut ist also in ihrer Entstehung wie auch Ausbildung ein von der Anwesenheit des Augenbeohers abhängiges Organ. Auf den Zusammenhang von Keimdrüsen und Gestaltung der sekundären Geschlechtscharaktere sei nur hingewiesen. Wir können also die seitherige, sog. deskriptive Morphologie in die Kategorie der .Seins"wissenschaften setzen. Demgegenüber ist die kausale Morphologie die „Werdens"wissenschaft. Sie sucht nach den Ursachen, die jene Zwangsläufigkeit in der Entwicklung, im Werden bedingen; sie lehrt uns. daß jene Ursachen in der befruchteten oder unbefruchteten (Parthenogenesis) Eizelle bereits determiniert sind und als Faktoren kontinuierlich bis zur vollendeten Differenzierung wirken. Sie zeigt auch die mannigfaltigen Wechselwirkungen der Teile aufeinander - ist dieses doch mit eingeschlossen im Begriffe der kausalen Erforschung. Aber nicht allein die' Ursachen der Formbildung will die Em. ermitteln, sondern auch die durch diese bedingten Prozesse, die zu den Gestaltungen führen. Sämtliche sichtbaren wie unsichtbaren Bewegungsvorgänge gilt es kausal darzulegen, die in der befruchteten Eizelle anheben und bis zum ausdifferenzierten, vielzelligen Organismus führen. Damit erstrebt sie gleichsam eine grandiose Physikochemie der Form des Organismus. Die Ueberzeugung waltet dabei vor, daß das gesamte Entwicklingsgeschehen mechanistisch auflösbar ist, da $\beta$ dieselben Energieformen und substanziellen Elemente in den Verlauf des or ganischen Werdens eingehen, die sich unserer Erkenntnis im Anorganischen dargeboten haben und dort noch darbieten werden, daß im Verlaufe der Organismendifferenzierung alle Vorgänge in ihren Elementarprozessen nicht qualitativ eigenartig sind, nicht andersartig als die das Gebiet der Physik und Chemie beherrschenden, sondern da $\mathrm{B}$ allein ihre Verkettung eigenartig ist. daß sie sowohl als die Größenordnung der sich abspielenden Reaktionen die größten Komplikationen in der Erforschung des Organischen bedingen. Aus dieser kausal-mechanistischen Interpretation der Entwicklungsvorgänge leitet sich der Name ,Entwicklungsmechanik“ ab.

Um diesen hat sich ein vielfältiger Streit erhoben, da den meisten Biologen seinerzeit und zum Teil auch noch heute bei -,,mechanik" allein die Vorstellung grober Massenbewegungen vorschwebte oder noch anderes, für unseren Fall Unzutreffendes. Nicht um eine Kinematik der Aufbauvorgänge handelt es sich, sondern um eine Kinetik. Roux, der gemeinsam mit $R$. Heidenhain jene Bezeichnung schuf, stellte sich auf den Standpunkt $K$ ants, der jede kausal eingestellte Wissenschaft als allein mechanistisch interpretierbar bezeichnete. Der von anderer Seite vorgeschlagene und auch bis heute beibehaltene Name Entwicklungsphysiologie (Driesch, Herbst, Loeb u. a.) trifft nicht das Richtige; denn er kann zu Verwechslung führen mit der Physiologie der Entwicklung. Zudem beschäftigt sich die Physiologie mit den Funktionen, die den Betrieb des Organismus oder seiner Teile aufrechterhalten, nicht aber mit denen, die ihn aufbauen, gestalten. Sie nimmt vielmehr in weitestem Sinne die Form als gegeben an und als stationär. Veränderungen kennt sie nur insofern, als es sich um reversible 7 ihrem Ausgangspunkt wieder zurückkehrende Bewegungen. handelt (Kontraktion, Sekretion usw.). Ferner ist die Physiologie durchaus nicht in allen $Z$ weigen kausal gerichtet, enthält vielmehr weite Gebiete rein deskriptiven Charakters. Driesch, der die Bezeichnung Entwicklungsphysiologie bevorzugt, steht allerdings, auch der prinzipiellen kausal-mechanistischen Interpretation ablehnend gegenüber $\mathrm{Da}$ eine große Reihe von Erscheinungskomplexen gegenwärtig in dieser Weise sich noch nicht auflösen läßt, dieser Forscher übrigens einer der scharfsinnigsten und erfolgreichsten der neuen Wissenschaft - ferner der Ansicht ist, da $B$ uns dieses auch in Zukunft nicht gelingen wird. stellte er sich auf vitalistischen Standpunkt, schreibt der zeit- und raumlosen und daher wissenschaftlich nicht faßbaren Entelechie die bewußt zwecktätig den Organismus formende Kraft zu. Der. Physiologe wird sich hiermit aber kaum einverstanden erklären. So wenig wie in der Chemie spielt in der Phvslologie jene rätselhafte „Kraft" auch nur noch die geringste Rolle.

Die Zielsetzung bestimmt in weitem Maße die Methoden einer Wissenschaft. Auch hierin war Roux der Hauptförderer der Em. Will man die Abhängigkeit irgendeiner Erscheinung von inneren oder äußeren Ursachen notwendig und hinreichend bestimmen, so müssen vor allem 'jene Erscheinungen, die es zu erklären gilt, klar und scharf erfaßt werden. Dies führte dazu, daß gewisse, seit langem gebräuchliche, aber in ganz verschiedener Weise verstandene Entwicklungsvorgänge neben noch anderen eine präzise Fassung erhielten und damit zu eindeutig definierten Begriffen wurden, wie sie jeder exakten Wissenschaft unentbehrlich sind, wie solche im Fundamente jeder exakten. Wissenschaft ruhen (Kraft, Druck, Arbeit, lon usw. in Physik und Chemie). So schuf Roux die Begriffe der Neoenigenesis und Neoevolutio, den Reizbegriff sonderte er von dem der Auslösung, den des Gesetzes von dem der Regel u. a. m.

Die Ursachen des Entwicklungsverlaufes bieten sich aber dem Forscher durchaus nicht so ohne weiteres dar. Sie sind vielmehr in der Regel von großer Kompliziertheit, stellen sich als "Ursachenkomplexe" ( $\mathrm{R}$ ou $\mathrm{x}$ ) dar. Ist es nicht möglich, sogleich die einfachen Ursachen (Faktoren) zu ermitteln, so müssen jene Komplexe aufgesucht werden. Ein Beispiel hierfür bietet jene oben angeführte Verursachung der Linsenbildung. Hier stellt die Anlegung des Retinablattes an das Ektoderm einen solchen Ursachen'omplex dar. Be der Feststellung dieses Komplexes darf es aber nicht bleiben. Vielmehr sind diese dann weiterhin Gegenstand der Forschung, indem man versucht, in sie einzudringen, um sie in weniger komplizierte zu zerlegen. Diese Zerlegung geschieht ataf dem Wege der Analyse sie ist eine gedankliche. So hatte sich im Falle der Linsenbildung als erstes Ergebnis gefunden, da $B$ der Augenbecher dazu notwendig 
war, und erst weiterhin stelle sich heraus, daB von diesem Komplex das Retinablatt, der engere, die Bildung bedingende war. Diese Analyse ist aber von der elementaren der deskriptiven. Morphologie dadurch unterschieden, daß sie eine kausale zu sein hat. Der Weg ist also der, daB von den komplizierten Ursachenverbindungen durch immer weiter fortgesetzte Auflösung in einfachere vorzudringen ist, bis man zu ganz einfachen gelangt, die möglichst anorganische sein sollen. Nur in relativ wenigen Fällen gelingt es, sogleich einfache Faktoren zu ermitteln - wo dies möglich ist, wird noch zu erörtern sein - und von diesen einfachen zu den zusammengesetzten Ursachen fortzuschreiten. Demgegenüber ist es von Bedeutung, festzustellen, daß die Em. an diesen Weg nicht gebunden ist, daß sie vielmehr '- wie dargelegt - die Möglichkeit - besitzt, den entgegengesetzten zu beschreiten, der von den komplizierten zu den einfachen führt. Diese kausale Analyse bildet einen der wesentlichsten methodologischen Bestandteile der neuen Morphologie. Hat man nun durch gedankliche Analyse eine Reihe von Ursachen für das Zustandekommen einer Erscheinung aufgefunden, so erhebt sich die Frage nach der Bedeutung jedes einzelnen Faktors unter ihnen. Diese kann dann dadurch festgelegt werden, daB man diesen Faktor nach Größe, Richtung usw. variiert, während alle anderen konstant gehalten werden. Will man etwa die Verkettung zweier oder mehrerer Ursachen ermitteln, so kombiniert oder variiert man diese und gelangt so zu exakten kausalen Ergebnissen. Alles dieses ist aber nur möglich unter Vornahme von Eingriffen in den normalen Entwicklungsverlauf. Die gedankliche Analyse allein vermag uns keine Ergebnisse zu liefern, denn es bedarf der Bestätigung, daß jene Analyse richtig und nicht irrig war. Diese Bestätigung nach der kausal-analytischen Vorarbeit liefert aber das Experiment. Dieses ist das zweite wichtige Forschungsmittel der Em. Es geht aus dem Dárgelegten klar hervor, daß die kausale Morphologie ein Experiment besonderer Art fordert. Es ist nicht wahllos und von ungefăhr vorzunehmen, vielmehr erfolgt es erst auf Grund der kausalen Analyse oder in Gemeinschaft mit ihr, es hat ein kausal-analytisches Experiment zu sein. Das ist als wesentlich festzuhalten; denn durch diesen geschilderten Charakter unterscheidet es sich grundsätzlich von dem deskriptiven Experiment, das nicht nach vorangegangener Analyse vorgenommen wurde und dessen Ergebnis dann auch nicht kausal ausgewertet wird und kausal auswertbar ist. Ein solches deskriptives Experiment wurde z. B. an der Blastula vorgenommen. Durch Anstiche schuf man Marken, an denen man dann später auf höherer Differenzierungsstufe ablesen konnte, welche Substanzumlagerungen im Differenzierungsvorgang stattgefunden hatten. Die "Experimentelle Zoologie" bediente sich schon vor Roux dieses Forschungsmittels und tut es auch gegenwärtig noch. Der prinzipielle Unterschied zwischen beiden Wissenschaftszweigen liegt eben in der Art der Verwendung des Versuches. Das kausal-analytische Experiment charakterisiert in.der dargelegten Weise die Em. Die Experimentelle Żoologie dagegen stellt allein die Erscheinungen an Organismen und ihren Teilen fest, die sich nach Eingriffen physikatischer oder chemischer Art ergeben. Sie unterläßt eine kausale Analyse. Gleichwohl gibt es hier naturgemä $B$ Grenzfälle, Fälle, wo aus dem deskriptiven Experiment sich ebenfalls ursächliche Feststellungen ableiten lassen. Aber das bleiben bestimmite, einfach gelagerte Grenzfälle, die den grundsätzlichen Unterschied beider Disziplinen nicht verwischen können. Von mancher Seite sind indes Sinn und Bedeutung des Experiments in der Morphologie abgelehnt worden, da durch Eingriffe in den "Werde"gang des Organismus Bedingungen geschaffen werden, die dem normalen Entwicklingsgang gänzlich fremd sind, die also pảthologische darstellen, aus denen man naturgemä $\beta$ nichts für den normalen Gestaltungsproze $\beta$ Gültiges folgern dürfe. Ohne näher auf diese Prinzipienfrage hier einzugehen, muß doch festgestellt werden, daß dieser Einwand auf einem Irrtum beruht oder auf Unkenntnis der Verhältnisse in bezug auf die Em. Diese läßt ja ausdrücklich nur das kausal-analytische Experiment $z u, d . h$. nur dasjenige, das sich ergab aus der vorangegangenen gedanklichen Analyse einer Erscheinung in thre ursächlichen Elemente. Habe ich einen Faktor gedanklich als verursachenden angenommen, so ist seine bloße Variation - natlirlich nur in sinngemäßer Weise! - ein Eingriff, durch den der normale Gestaltungsverlauf durchaus nicht der Art nach geändert wird. vielmehr "zwingen wir gleichsam die personifiziert gedachte sog. Natur; auf unsere Fragen nach ihrem Wirken Antwort zu geben" (Ro u x). Die wichitigste Kontrolle aber bleibt das Ergebnis; denn stets muB es in einem bestimmten, diskutierbaren Verhältnis zum Eingriffe stehen, wenn überhaupt ein SchluB von Folge auf Ursache vollziehbar sein soll. Der Eingriff als solcher ist ein atypischer Vorgang. Sein Effekt muß aber mit dem . Typus in einer Beziehung stehen Durch die analytisch-experimentelle Methode ist die Em. in -die Reihe der exakten Naturwissenschaften getreten. Freilich muß man zugestehen, daß die Em. bisher den höehsten möglichen Grad-der Exaktheit, den Physik, Chemie und zum Teil auch Physiologie bereits besitzen, noch nicht hat erreichen können, jenen Grad der Exaktheit, der auf quantitativen Resultaten beruht. Bis heute muß sich die kausale Morphologie noch in überwiegendem Maße mit rein qualitativen Ergebnissen begnügen. Eine große Reihe von Nachteilen bringt dieses mit sich, vielleicht aber ist die Zeit nicht fern, wo mit weiterem Eindringen physikalisch-chemischer Arbeitsmethoden in die Morphologie quantitative Bestimmungen prinzipiell Einzug halten in diese Wissenschaft. 